\title{
Electrons Emitted from 33-TeV Pb Ions During Penetration of Solids
}

\author{
C. R. Vane, U. Mikkelsen, ${ }^{a}$ H. F. Krause, ${ }^{*}$ S. Datz, ${ }^{*}$ P. Grafström, \\ H. Knudsen, ${ }^{a}$ S. Møller, ${ }^{a}$ E. Uggerhøj, ${ }^{a}$ \\ C. Scheidenberger, ${ }^{c}$ R. H. Schuch, ${ }^{d}$ and Z. Vilikazie \\ *Physics Div., Oak Ridge National Laboratory, P.O. Box 2008, Oak Ridge, TN 37831 -6377 USA

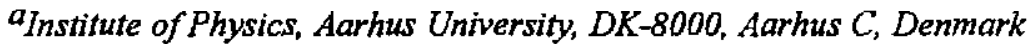 \\ $b_{C E R N}$ SPSSLL Division, CH-1211, Geneva 23, Switzerland \\ ${ }^{c}$ Gesellschaft far Schwerionenforschung mbh, Planckstrasse 1, D-64291, Darmstadt, Germany \\ $d_{\text {Atomic Physics Department, Stockholm University, Frescativagen } 24 .}$ \\ S-104 05, Stockholm 50, Sweden \\ eSchonland Research Centre for Nuclear Sciences, Johannesburg, 2050 Gauteng, South Africa
}

\section{INTRODUCTION}

At ultrarelativistic energies, ionization cross sections exceed electron capture cross sections by several orders of magnitude $(1,2)$. Effectively, all electrons transferred to a highly relativistic heavy ion moving in a solid or gaseous target medium are stripped in a relatively short distance. Above $\sim 20 \mathrm{GeV} /$ nucleon, the principal mechanism for electron capture is from pair production (ECPP) (2). The total cross sections for ECPP are technically important for making reliable predictions of operating limitations for relativistic heavy-ion colliders, e.g., RHIC and LHC (3). In ECPP, it is expected that $\sim 30 \%$ of capture proceeds to excited states of the capturing ion. Some of these relatively weakly bound electrons are radiatively long-lived and easily lost in secondary collisions in solid targets, making measurements of their contributions to total capture experimentally difficult. Electrons lost from high-energy ions in collisions with target atoms form a cuspshaped spectral peak in the forward direction in the laboratory frame centered at the velocity of the moving ion (4-5). The shape of this electron loss to projectile continuum (ELC) peak has been shown $(5,6)$ to depend on the initial atomic bound state from which the electron is ionized. We have measured and compared ELC electrons from direct ionization of hydrogenlike $33-\mathrm{TeV} \mathrm{Pb}^{81+}$ (1s) ions (Lorentz factor $\gamma=168$ ) in Al with similar data for electrons created by ECPP for bare $\mathrm{Pb}^{82+}$ ions in $\mathrm{Au}$ - followed by ionization. Both measured ELC peaks are narrow in momentum and angle and very similar in shape.

For fully-stripped 33-TeV $\mathrm{Pb}^{82+}$ ions, production of ELC electrons requires a two-step, double-collision process. In the first step, the $\mathrm{Pb}^{82+}$ ion interacts electromagnetically with a target atom forming an electron-positron pair. The

"The subrit tee manuscrpt has been authored by acontractor of the U.S. Government under Contract Na. DE-ACO5-960R22464. Accordingly, the U.S.Government retains a nonexeclusive, his cortribation, or allow other to do so, for U.S. Government purposes. 
electron formed in atomic bound states of the resulting $\mathrm{Pb}^{81+}$ ion through ECPP is carried along at the projectile velocity. In a second step, the $\mathrm{Pb}^{81+}$ ion is ionized in a long-range collision with another target atom. The low-energy ionized projectileframe electrons, appear in the lab frame at $\gamma=168$, or $\sim 86 \mathrm{MeV} / \mathrm{c}$. Free-pair electrons form an underlying continuum covering several hundreds of $\mathrm{MeV} / \mathrm{c}$. Corresponding positrons are emitted as free particles in either case.

A schematic of the apparatus used is shown in Fig. 1. Either hydrogenic or fullystripped 33-TeV Pb ions from the CERN SPS accelerator were passed through thin foil targets in vacuum. Electrons collimated to $0.55^{\circ}$ half-angle to eliminate contamination from direct knock-on electrons were deflected in a uniform magnetic analyzing field of $\sim 0.1 \mathrm{~T}$, and passed through two $2 \mathrm{D}$ position-sensitive drift chamber detectors located outside the analysis field. The two detector coordinate positions for coincident hits were used to calculate the trajectory for each electron and determine both the corresponding ejection angle and, from the horizontal deflection in the magnetic field, the momentum. Ions were counted in a fast scintillator detector for timing and coincidence. Free positrons were also detected in a large scintillator counter.

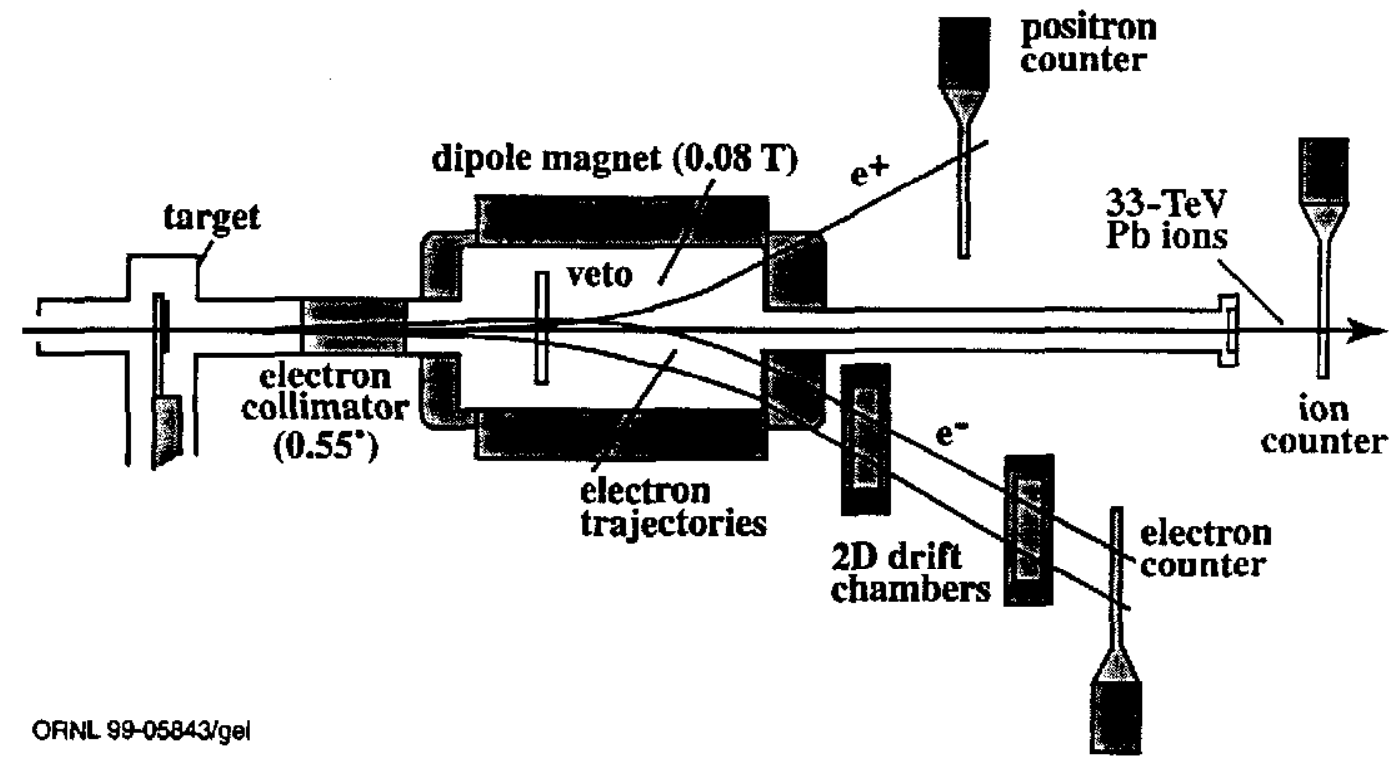

Figure 1. Experimental apparatus. Magnetic spectrometer for zero-degree ELC electrons.

The ELC electrons were momentum dispersed by the uniform magnetic analyzing field only in the horizontal plane. The vertical angle distributions, as shown in Fig. 2, obtained from trajectory analysis of the two 2D drift chamber hit positions, agree with calculations for a combination of detector position resolution and accumulated angular broadening due to multiple Coulomb scattering in the targets, the aluminum exit window from the vacuum chamber, and from air between the 
window and the detectors. This vertical distribution for each target was used to correct the horizontal position distribution data for instrumental broadening to obtain the ELC momentum distributions.

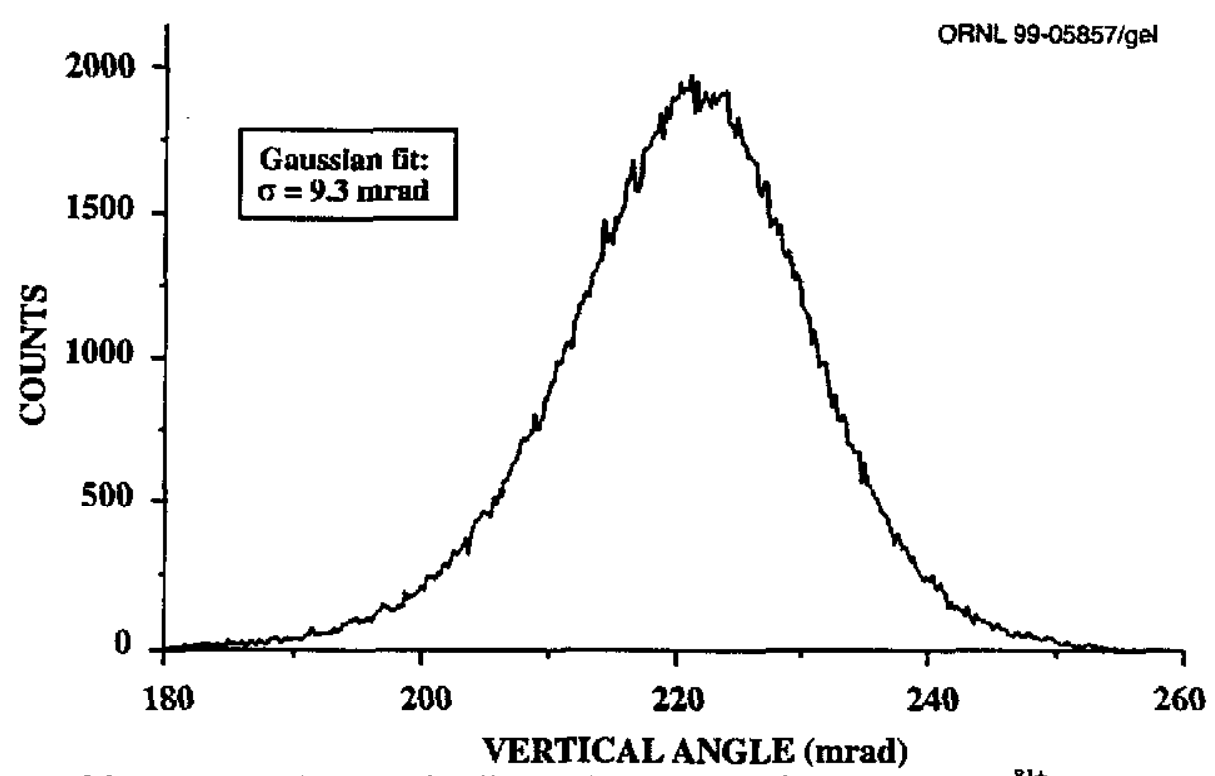

Figure 2. Vertical angular distribution for ELC electrons from $\mathrm{Pb}^{81+}+\mathrm{Al}$.

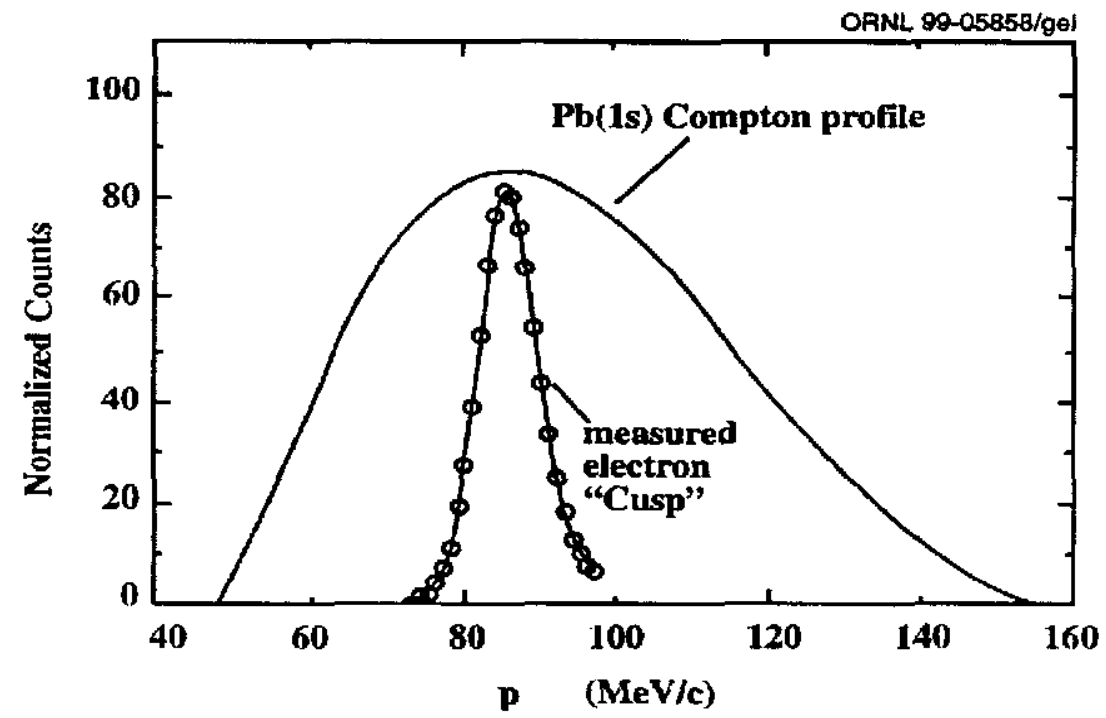

Figure 3. Measured $\mathrm{Pb}^{81+}(1 \mathrm{~s})+\mathrm{Al}$ ELC momentum distribution compared with Compton Profile.

Figure 3 shows a comparison of the measured ELC 'cusp' electron momentum distribution for 33- $\mathrm{TeV} \mathrm{Pb}^{81+}(1 \mathrm{~s})$ ionized in an $\mathrm{Al}$ target with a simple $\mathrm{Pb}(1 \mathrm{~s})$ hydrogenic Compton profile mapped into the laboratory frame. The assumed angular emission distribution in the projectile frame is dipole, i.e., $\sim \sin ^{2}\left(\theta_{\text {proj }}\right)$. The comparison is made simply to indicate the relatively narrow shape of the ELC 
peak. In Fig. 4, we show an expanded view of the measured ELC electron momentum distribution from $33-\mathrm{TeV} \mathrm{Pb}^{81+}(1 \mathrm{~s})$ ions passing through a $77-\mathrm{mg} / \mathrm{cm}^{2}$ Al foil. The figure also shows the maximum width 'cusp'-shaped peak consistent with the measured momentum profile, convoluted with the overall instrument function which was taken directly from the measured vertical electron distribution. The fitting 'cusp'-shape was chosen somewhat arbitrarily to be the 'Dettmann' cusp shape, which is symmetric and proportional to

$$
\sqrt{\left(p-p_{0}\right)^{2}+p_{0}^{2} \theta^{2}}-\left|p-p_{0}\right|
$$

where here $\theta_{0}$ is simply a fitting parameter, $p$ is the electron momentum, and $p_{0}$ is the electron momentum at the projectile velocity $(86 \mathrm{MeV} / \mathrm{c})$.

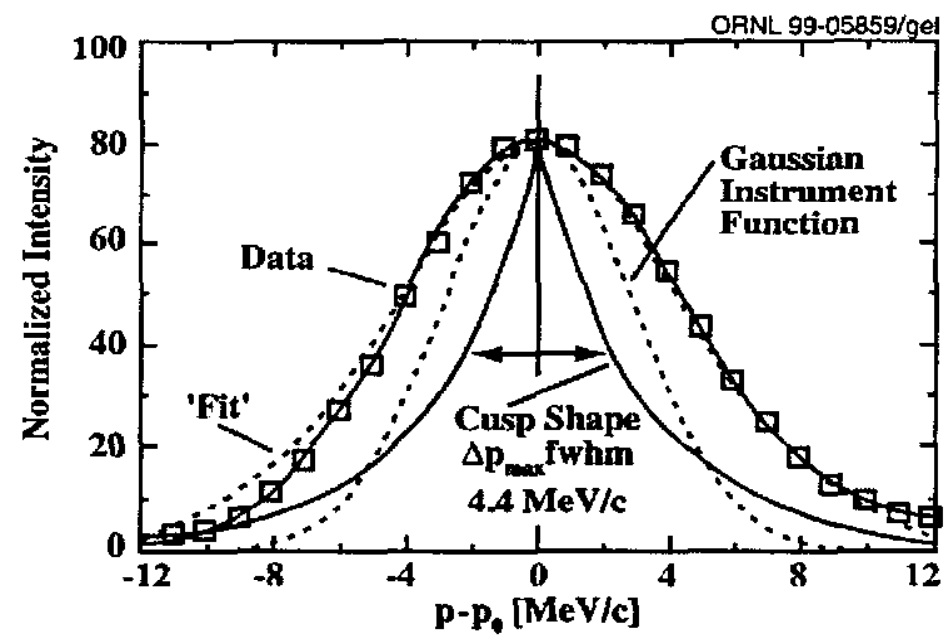

Figure 4. Measured $\mathrm{Pb}^{81+}(1 \mathrm{~s})+\mathrm{Al}$ ELC momentum distribution and maximum-width symmetric cusp-shaped spectrum consistent with the data.

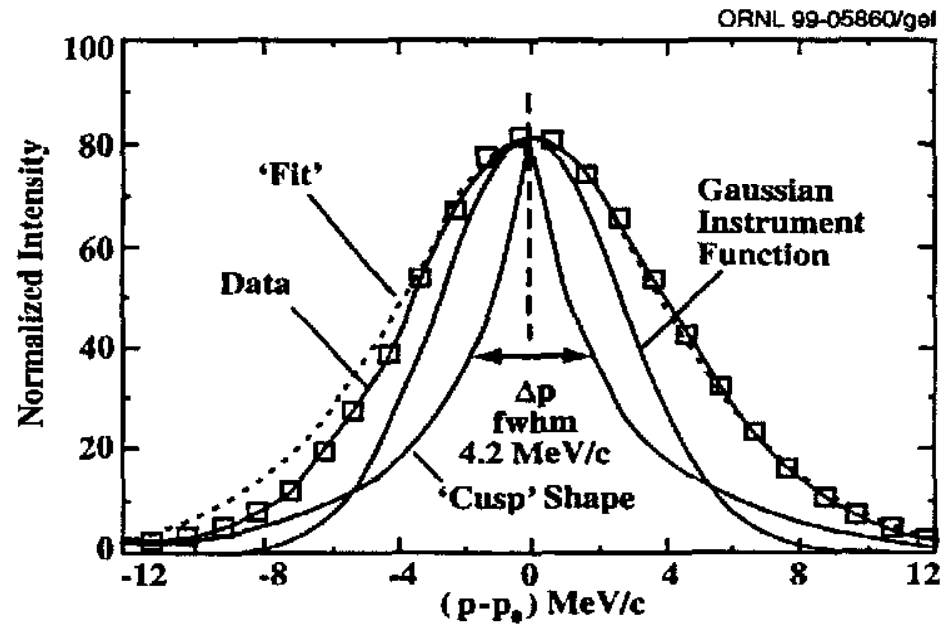

Figure 5. $\mathrm{Pb}^{82+}+\mathrm{Au}$ momentum distribution and maximum-width cusp-shaped spectrum. 
Figure 5 shows the measured ELC electron momentum distribution from $33-\mathrm{TeV}$ $\mathrm{Pb}^{82+}$ ions passing through a $17-\mathrm{mg} / \mathrm{cm}^{2}$ Au foil. The figure also shows the maximum width 'cusp'-shaped peak. For fully-stripped ions, the ELC electrons must come from ECPP ( $>95 \%)$, followed by ionization. The 'cusp'-shape observed should then somewhat represent ELC from the states populated through ECPP. We see essentially no evidence for a significant population of excited states leading to a narrower peak component than observed for ionized $\mathrm{Pb}^{81+}(1 \mathrm{~s})$ ions.

\section{CONCLUSIONS}

We have measured electrons ejected in the forward direction from bare- and oneelectron 33-TeV Pb ions passing through thin foils of $\mathrm{Al}$ and $\mathrm{Au}$. We observed spectral peaks centered at a momentum equivalent to the velocity of the projectile, and attribute these electrons to ELC. From previous theoretical studies, the ELC 'cusp' shape should reflect somewhat the origin of the electron released from the projectile - ls electrons leading to broader cusp shapes than more highly excited n-state electrons. The observed ELC peaks are very narrow even for loss from ground-state ions. The peaks are so narrow that the measured widths arise mainly from instrumental broadening effects - especially multiple Coulomb scattering. These broadening effects are directly and independently measurable in the experiment. Correction of the electron momentum distributions for measured broadening leads us to conclude that the ELC cusps are intrinsically very narrow, and essentially the same for loss from ground-state ions and from ions populated by electron capture from pair production (ECPP). Analysis is continuing to attempt to place limits on the fraction of any excited-state population consistent with these observations.

\section{ACKNOWLEDGMENTS}

Authors (HFK,CRV,SD) gratefully acknowledge support by the USDOE, Office of Basic Energy Sciences, Division of Chemical Sciences, under Contract No. DE-AC05-96OR22464 with Lockheed Martin Energy Research Corporation. HK acknowledges the support of the Danish Natural Science Research Council.

\section{REFERENCES}

1. Eichler, J. and Meyerhof, W. E., Relativistic Atomic Collisions, San Diego: Academic Press, 1995.

2. Krause, H. F. et al., Phys. Rev. Lett. 80, 1190 (1998).

3. Grafström, P. et al., CERN Report SL-99-033EA (1999).

4. Breinig, M. et al., Phys. Rev. A 25, 3015 (1982).

5. Burgdörfer, J. et al., Phys. Rev. $A$ 28, 3277 (1983).

6. Takabayashi, Y. et al., contributed paper TU120, ICPEAC XXI, Sendai, Japan, 1999. 